\title{
Comparison of rapamycin and methylprednisolone for treating inflammatory muscle disease in a murine model of experimental autoimmune myositis
}

\author{
JUAN KANG $^{1}$, DONGYUN FENG ${ }^{1}$, FENG YANG ${ }^{1}$, XIAOJIA TIAN $^{1}$, WENJUAN HAN ${ }^{1}$ and HONGGE JIA ${ }^{1,2}$ \\ ${ }^{1}$ Department of Neurology, Xijing Hospital, The Fourth Military Medical University, Xincheng, Xi'an, Shaanxi 710032; \\ ${ }^{2}$ Department of Neurology, Shenzhen Hospital, Southern Medical University, Shenzhen, Guangdong 518034, P.R. China
}

Received April 1, 2019; Accepted December 9, 2019

DOI: $10.3892 /$ etm. 2020.8716

\begin{abstract}
Idiopathic inflammatory myopathies (IIMs) are a group of autoimmune inflammatory muscle diseases. Rapamycin has been shown to ameliorate inflammation and improve muscle function in a mouse model of experimental autoimmune myositis (EAM). In the present study, the therapeutic effect of rapamycin was compared with methylprednisolone (MP) on EAM. Mice were injected with myosin for 10 days to induce EAM and were subsequently treated with rapamycin $(1.5 \mathrm{mg} / \mathrm{kg}), \mathrm{MP}(40 \mathrm{mg} / \mathrm{kg})$ or placebo (DMSO) for 14 days. The rapamycin-treated group exhibited significantly decreased severe inflammation and improved muscle strength compared with the MP-treated group. The plasma transforming growth factor- $\beta$ (TGF- $\beta$ ) concentration in the rapamycin-treated group was significantly higher compared with the placebo group. However, both treatment groups exhibited significantly lower plasma interleukin-10 levels compared with the placebo group. Moreover, splenic regulatory $\mathrm{T}$ cell frequency in both the rapamycin- and MP-treated animals was significantly lower than that in the animals of the placebo group. Rapamycin showed better immune suppressive effects than MP in this model of EAM, and these effects were likely to be mediated by the TGF- $\beta$ signaling pathway.
\end{abstract}

Correspondence to: Dr Hongge Jia, Department of Neurology, Xijing Hospital, The Fourth Military Medical University, 127 Changle West Road, Xincheng, Xi'an, Shaanxi 710032, P.R. China E-mail: jiahg@fmmu.edu.cn

Abbreviations: IIMs, idiopathic inflammatory myopathies; EAM, experimental autoimmune myositis; MP, methylprednisolone; DM, dermatomyositis; IBM, inclusion body myositis; TGF- $\beta$, transforming growth factor- $\beta$; IL-10, interleukin-10

Key words: rapamycin, IIMs, EAM, Treg cells, TGF- $\beta$

\section{Introduction}

Idiopathic inflammatory myopathies (IIMs) are a group of inflammatory muscle diseases that share similar clinical presentations, such as muscle weakness (1-5). IIMs include dermatomyositis (DM), polymyositis (PM) and inclusion body myositis (IBM). The pathogenesis of the individual conditions may differ. However, inflammatory cell infiltration into skeletal muscles is common across these disorders. In patients with IIMs, helper T cells (CD4 T cells) and cytotoxic T cells (CD8 T cells) have been shown to invade muscle fibers (6). Autoantibodies in the endomysium, perimysium, perivascular areas and blood have also been reported $(7,8)$.

Despite years of research, the underlying mechanisms of IIMs are not fully understood. As a result, there are no targeted immunotherapies for these disorders. Currently, patients with IIMs are treated with immune-suppressive corticosteroids, such as short-term methylprednisolone (MP) or long-term prednisone. However, treatment with corticosteroids is not ideal, since long-term use of prednisone can result in severe loss of muscle strength, which is contradictory to the treatment goal (1). Immunoglobulin infusion is another treatment option, but it is costly and is typically reserved for patients who are refractory to corticosteroids or have trouble swallowing, and for patients for whom immunosuppressants are contraindicated due to comorbid conditions. Thus, immunomodulatory therapies become of interest, since they can improve the conditions of the disease and decrease the adverse effects associated with corticosteroids $(1,9,10)$.

Rapamycin inhibits immune cell proliferation, thereby suppressing the immune response, and has been shown to be effective in treating various inflammatory conditions (11-17). Rapamycin possibly suppresses the immune response by increasing the frequency of regulatory $\mathrm{T}$ (Treg) cells, a subset of $\mathrm{T}$ cells that modulates the immune system and has been postulated to prevent autoimmune disorders. Depletion of Treg cells leads to excessive proliferation of effecter T cells, which can lead to autoimmune diseases such as polyendocrinopathy, enteropathy and X-linked inheritance (18-20). By contrast, increased numbers of Tregs and Treg activity can protect individuals from autoimmune diseases (21). 
In order to study the mechanisms underlying IIMs and to test treatment efficacy, a murine model of experimental autoimmune myositis (EAM) was developed. EAM shares disease characteristics with PM, such as T cell infiltration of muscle fibers $(2,6)$. Allenbach et al (22) reported that the depletion of Treg cells aggravated EAM, whereas polyclonal Treg injection improved the condition. Furthermore, Prevel et al (23) found that rapamycin increased Treg cell frequency and showed beneficial effects in treating EAM. Therefore, rapamycin is a potential treatment option for myositis because it suppresses the immune system by increasing Treg frequency.

To date, no direct comparison between rapamycin and steroids for treating myositis has been made, to the best of our knowledge. In the present study a murine model of EAM was used to compare the efficacy of rapamycin and MP, and to further investigate the possible mechanism of action of rapamycin for treating EAM.

\section{Materials and methods}

Animals. A total of 24 female BALB/c mice (weight 15-17 g; age, 6-8 weeks) and 1 female guinea pig (weight, $480 \mathrm{~g}$; age, 12 weeks) were purchased from the Experimental Animal Center of The Fourth Military Medical University (Xi'an, China). All mice and guinea pigs were housed in the specific pathogen-free facility of the Experimental Animal Center at room temperature $\left(22 \pm 1^{\circ} \mathrm{C}\right)$ and $40-60 \%$ humidity with a 12/12 h light/dark cycle, and access to food and water ad libitum. Mice were anesthetized via an intraperitoneal injection of $10 \%$ chloral hydrate, at a dose of $3 \mathrm{ml} / \mathrm{kg}$ body weight (350 mg drug per $\mathrm{kg}$ body weight). Mice and guinea pigs were euthanized using $\mathrm{CO}_{2}$ asphyxiation (the flow rate of $\mathrm{CO}_{2}$ was about $10-30 \%$ of the chamber volume per minute), followed by cervical dislocation. The animals did not exhibit any evident signs of peritonitis following the administration of $10 \%$ chloral hydrate.

EAM model. EAM was induced using a previously published protocol (24). Briefly, skeletal muscle from a guinea pig was dissected, weighed and frozen at $-70^{\circ} \mathrm{C}$. The frozen muscle tissue was subsequently thawed and minced. The muscle tissue $(10 \mathrm{~g})$ was homogenized in $30 \mathrm{ml}$ homogenizing buffer $(0.3 \mathrm{M}$ $\mathrm{KCl}$ and $0.15 \mathrm{M}$ sodium phosphate; $\mathrm{pH} 6.5$ ) at $4^{\circ} \mathrm{C}$ and kept on ice for $1 \mathrm{~h}$. The homogenate was subsequently centrifuged at $12,000 \mathrm{x} \mathrm{g}$ for $30 \mathrm{~min}$ at $4^{\circ} \mathrm{C}$, and the supernatants were collected and filtered. The filtrates were diluted with cold Milli-Q-filtered water (5X the volume of the filtrates), and the resultant solution was then centrifuged at $7,000 \times \mathrm{g}$ at $4^{\circ} \mathrm{C}$ for $30 \mathrm{~min}$. The aggregated myosin, the pellet obtained following centrifugation, was resuspended in $0.5 \mathrm{M} \mathrm{KCl}$ and stored at $-70^{\circ} \mathrm{C}$.

To induce EAM, mice were subcutaneously injected with $100 \mu 1$ of $50 \%$ complete Freund's adjuvant (Sigma-Aldrich; Merck KGaA) containing $1.5 \mathrm{mg}$ myosin and $5 \mathrm{mg} / \mathrm{ml}$ Mycobacterium tuberculosis (BD Difco ${ }^{\mathrm{TM}}$ Adjuvants; BD Biosciences; cat. no. DF3114-33-8) in the left hind limb. Booster shots were administered at the tail base once a week for 2 weeks. Immediately after each booster shot, the mice were intraperitoneally injected with pertussis toxin
(500 ng in $200 \mu \mathrm{l}$ saline; Sigma-Aldrich; Merck KGaA). The myosin solutions were freshly prepared or stored at $-70^{\circ} \mathrm{C}$ for $<1$ month

A total of 10 days following the last injection of myosin, rapamycin $(1.5 \mathrm{mg} / \mathrm{kg}$ body weight) or MP $(40 \mathrm{mg} / \mathrm{kg}$ body weight) was administered intraperitoneally into the mice daily for 14 days. Mice in the placebo group received equal volumes of $3 \%$ DMSO (diluted in saline) via intraperitoneal injection. The mice were assessed for muscle strength on day 15 , following drug treatment, and tissues were then collected for subsequent analysis.

Inverted screen test. Muscle strength was assessed using the inverted screen test, as previously described $(21,24,25)$. Briefly, the mice were placed at the center of a circular wire mesh screen $\left(50 \mathrm{~cm}^{2}\right)$, composed of $1 \mathrm{~mm}$ diameter wire. The screen was immediately rotated to the inverted horizontal position for $3 \mathrm{sec}$, with the head of the mouse declining first. The screen was then held steadily $20 \mathrm{~cm}$ above a padded surface. The time for the mouse to fall from the mesh was recorded. Each mouse was assessed five consecutive times.

Histological grading of inflammation. Muscle tissue sections (10 $\mu \mathrm{m}$ thick) were randomly selected from each block and stained with hematoxylin and eosin. Briefly, samples were fixed with $10 \%$ neutral buffered formalin overnight at room temperature and embedded in paraffin. The paraffin sections, with a thickness of $10 \mu \mathrm{m}$, were deparaffinized and rehydrated with xylene and ethanol in gradient concentrations and stained with hematoxylin for $10 \mathrm{~min}$ at room temperature. After destaining with $10 \%$ acid ethanol for 5-10 sec and washing with water, the slides were stained with eosin for $30 \mathrm{sec}$ at room temperature and subjected to dehydration with ethanol and xylene. The slides were mounted with Permount ${ }^{\mathrm{TM}}$ mounting medium (Thermo Fisher Scientific, Inc.) and observed under a confocal microscope (LeicaDRM; Leica Microsystems Inc.) with 10x, $20 \mathrm{x}$ and 40x magnification. Inflammation in six muscle tissue sections was graded and expressed as a mean score (26-28), which was defined as follows: Grade 1, fewer than five muscle fibers involved; grade 2, a lesion involving 5-30 muscle fibers; grade 3 , a lesion involving a muscle fasciculus; and grade 4, diffuse extensive lesions. When multiple lesions were found in one section of muscle, 0.5 was added to the score. Five random fields of view per sample were assessed for inflammation scoring in a blinded manner by two independent trained pathologists.

Luminex assay. Plasma transforming growth factor- $\beta$ (TGF- $\beta$ ) and interleukin 10 (IL-10) levels were measured using the Luminex assay. Briefly, blood samples from mice were collected via the retro-orbital plexus under general anesthesia with $10 \%$ chloral hydrate $(350 \mathrm{mg} / \mathrm{kg})$, as described above, placed at room temperature for $30 \mathrm{~min}$, and then plasma was sampled after centrifugation at $10,000 \mathrm{~g} / \mathrm{min}$ at $4^{\circ} \mathrm{C}$ for $10 \mathrm{~min}$. The Luminex multiplex assays (cat. no. TGFB-64K-01 for the detection of TGF- $\beta$ and cat. no. MPXMCYTO-70K for the detection of IL-10; EMD Millipore) were performed according to the manufacturer's protocols. Samples were assessed in duplicate with the Luminex 200 IS System (Luminex Corporation). TGF- $\beta$ and IL-10 were identified and classified 
with the red laser, and their protein levels were quantified using the green laser. Following laser excitation, digital images of the bead array were captured and processed on a computer workstation. Standard curves and reports of unknown samples were prepared using the BeadView and MiraiBio software (Vigene Tech, Inc; version 3.1). The limits of sensitivity for the assay were $9.8 \mathrm{pg} / \mathrm{ml}$ for TGF- $\beta$ and $1.25 \mathrm{pg} / \mathrm{ml}$ for IL-10. All mice had detectable levels of these cytokines.

Flow cytometry. The composition of splenocytes was analyzed using flow cytometry. Fresh spleens were ground with the plunger of a $5 \mathrm{ml}$ sterile syringe in $\mathrm{PBS}$ at $4^{\circ} \mathrm{C}$, and the homogenate was then passed through a nylon mesh screen. The lymphocytes were separated in EZ-Sep ${ }^{\mathrm{TM}}$ Mouse $1 \mathrm{X}$ lymphocyte separation medium (Dakewe Biotech Co., Ltd.) and resuspended in flow cytometry staining buffer (PBS supplemented with $2 \%$ FBS; Thermo Fisher Scientific, Inc.). Splenocyte cell viability, as detected by trypan blue staining, was $>95 \%$.

Cells were washed once in flow cytometry staining buffer, and then resuspended to a density of $1 \times 10^{7}$ cells $/ \mathrm{ml}$. For surface staining, cells were incubated with FITC-labeled anti-mouse CD4 (clone RM4-5; cat. no. 11-0042-82; Thermo Fisher Scientific, Inc.) and allophycocyanin-labeled anti-mouse CD25 (clone PC61.5; cat. no. 17-0251-82; Thermo Fisher Scientific, Inc.) for $\sim 30 \mathrm{~min}$ at $4^{\circ} \mathrm{C}$. Appropriate isotype control antibodies (cat. no. 11-4321-80 and cat. no. 17-4301-82; Thermo Fisher Scientific, Inc.) were used to exclude nonspecific binding after washing, fixation and permeabilization of the cells. Cell fixation and permeabilization were conducted at $4{ }^{\circ} \mathrm{C}$ for $2 \mathrm{~h}$ with the eBioscience ${ }^{\mathrm{TM}}$ Mouse Regulatory $\mathrm{T}$ Cell Staining Kit \#2 (cat. no. 88-8118-40; Thermo Fisher Scientific, Inc.) following the manufacturer's instructions. Subsequently, the cells were stained intracellularly with phycoerythrin-labeled anti-mouse/rat Foxp3 (FJK16-second; eBioscience; Thermo Fisher Scientific, Inc.), following the manufacturer's instructions. The cells were then applied to a FACScan cytometer equipped with the CellQuest software (version 3.0, BD Biosciences) for analysis.

Statistical analysis. All data are presented as the mean \pm SD (from at least 3 repeats), unless otherwise noted. One-way analysis of variance followed by the post hoc least significant difference test was used to assess inter-group differences. $\mathrm{P}<0.05$ was considered to indicate a statistically significant difference. All analyses were performed using SPSS 16.0. software (SPSS, Inc.).

\section{Results}

General body condition assessment. A murine model of EAM was first established, which was induced by injections with myosin. All myosin-injected mice developed dull and untidy fur, lost weight, and became less active. Moreover, skin ulcers were found at the injection sites (Fig. 1A). The spleens of all myosin-injected animals were enlarged compared with the placebo animals (mean spleen length, $1.40 \pm 0.12 \mathrm{~cm}$ ), which indicated that inflammation was induced upon myosin injection. Subsequent treatment with
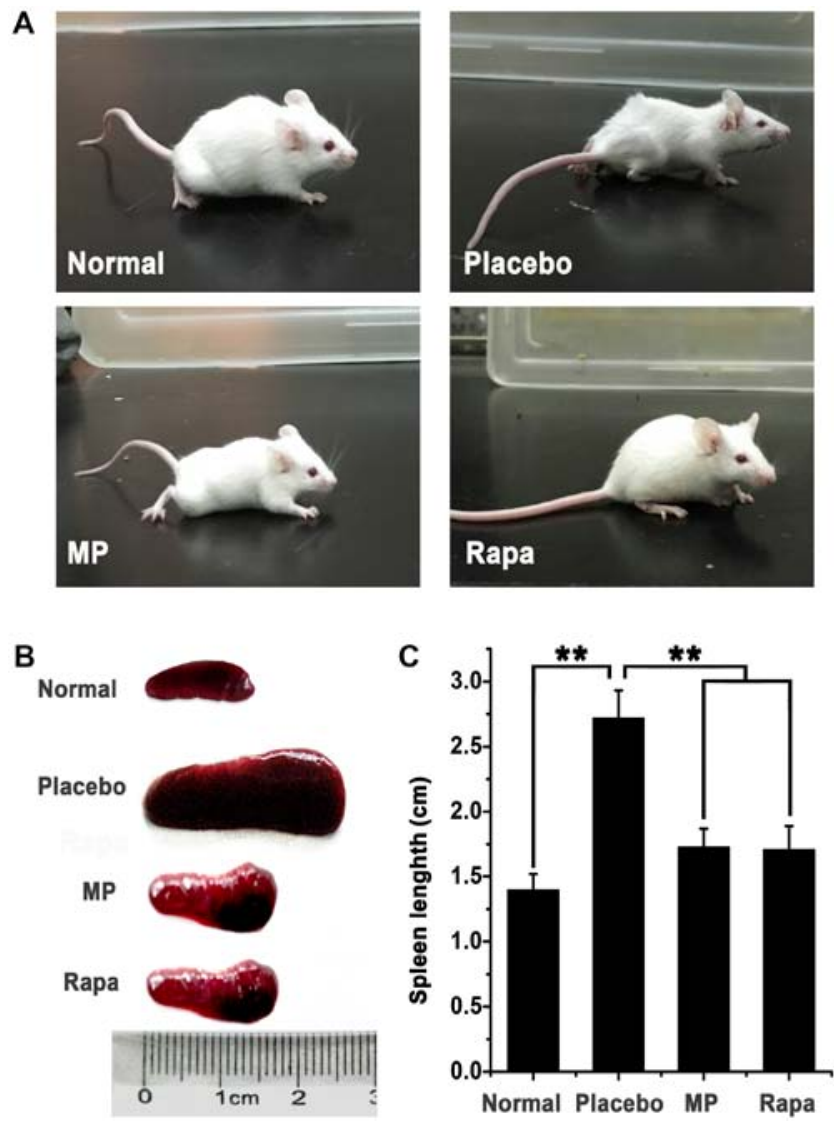

Figure 1. EAM model. (A) Representative images of mice at 15 days after initiation of the indicated treatments. Normal, control normal mice without myosin injection; placebo, EAM mice treated with vehicle; MP, EAM mice treated with MP; Rapa, EAM mice treated with Rapa. (B) Representative images of dissected spleens from each group. (C) Summary of spleen sizes in each group. At 15 days after the initial drug treatments, spleens from the MP- and Rapa-treated mice were significantly smaller compared with those from the placebo group. $\mathrm{n}=6$ for each group. ${ }^{* *} \mathrm{P}<0.01$. EAM, experimental autoimmune myositis; MP, methylprednisolone; Rapa, rapamycin.

either MP or rapamycin improved the general body condition of the animals and resulted in less marked enlargement of the spleen compared with the placebo group (Fig. 1B). The mean spleen length was $2.72 \pm 0.21 \mathrm{~cm}$ in the placebo group compared with $1.73 \pm 0.14 \mathrm{~cm}$ in the MP-treated group and $1.71 \pm 0.18$ in the rapamycin-treated group. Spleens in the drug-treated groups were significantly smaller compared with those in the placebo group $(\mathrm{P}<0.01$, treatment group vs. placebo group), suggesting that drug treatments effectively ameliorated inflammation in mice. Spleen sizes between the rapamycin-treated mice and MP-treated mice did not differ significantly $(\mathrm{P}>0.05$; Fig. $1 \mathrm{C})$.

Histological grading of muscle inflammation. Muscle inflammation was scored using a 1-4 grading system, with the score of 1 being the least severe (Fig. 2A-C). The inflammation score was $2.22 \pm 0.12$ for the placebo group, $1.72 \pm 0.16$ for the MP-treated group $(n=6 ; \mathrm{P}<0.01$ vs. placebo group) and 1.28 \pm 0.19 for the rapamycin-treated group $(n=6$; $\mathrm{P}<0.01$ vs. placebo group) (Fig. 2D). Furthermore, the inflammation score of the rapamycin-treated group was significantly lower compared with that of the MP-treated group $(\mathrm{P}<0.01)$. 

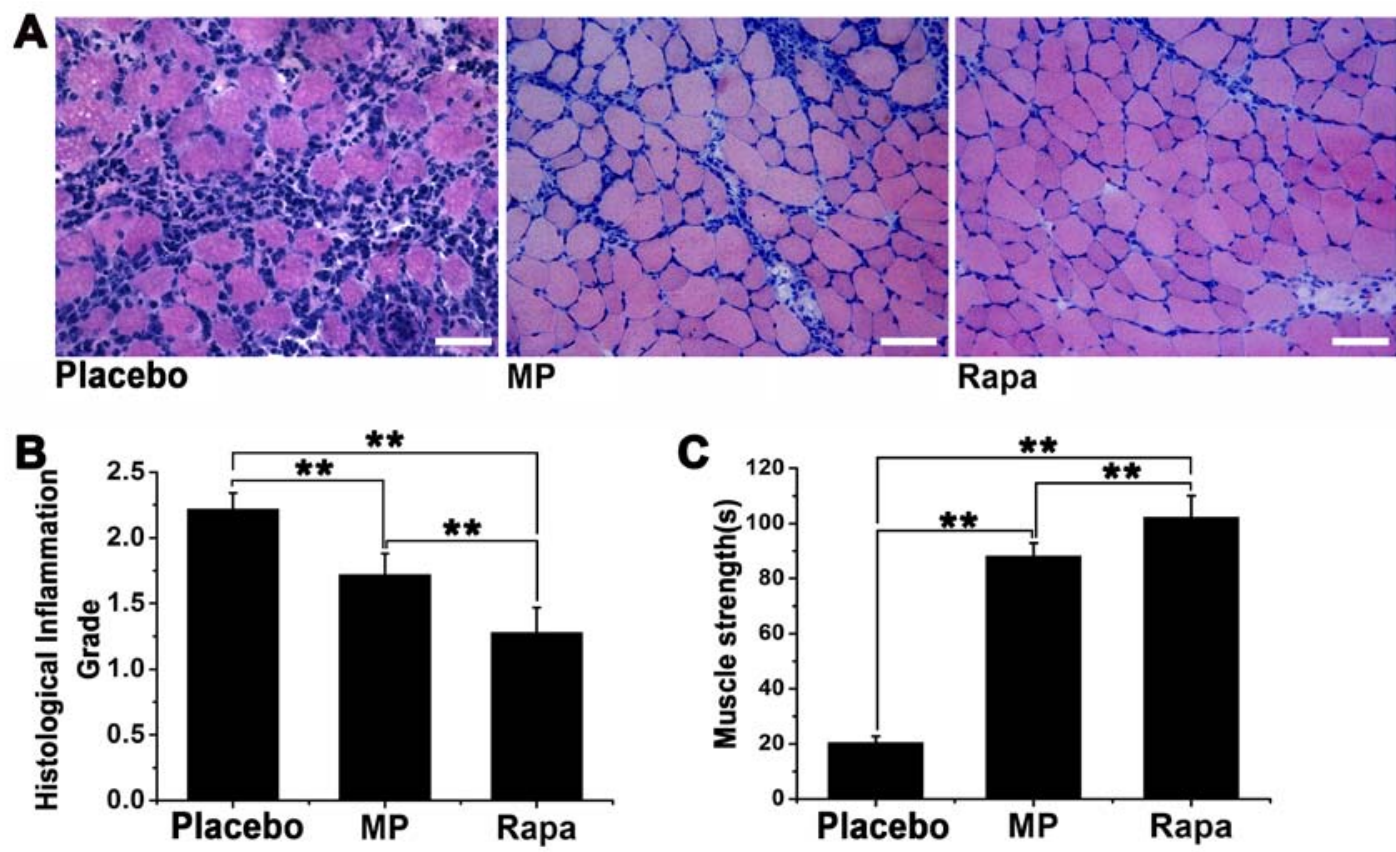

Figure 2. Compared with both the MP and placebo groups, treatment with rapamycin significantly alleviates inflammation and improves muscle strength in EAM mice. (A-C) Representative muscle sections from each group at 15 days after initiation of the indicated treatments. Scale bar, $50 \mu \mathrm{m} .{ }^{* *} \mathrm{P}<0.01$. MP, methylprednisolone; EAM, experimental autoimmune myositis; Rapa, rapamycin.

Muscle strength was assessed by measuring the time-to-fall in the inverted screen test for each mouse. Our preliminary experiments indicated that under normal circumstances, a healthy BALB/c mouse (weight 15-17 g, age 6-8 weeks) can stay on the inverted screen for $>30$ min before falling (data not shown). By contrast, the average time-to-fall was $20.50 \pm 2.27 \mathrm{sec}$ for the placebogroup, $88.13 \pm 4.77 \mathrm{sec}$ for the MP-treated group ( $\mathrm{P}<0.01$ vs. placebo group), and $102.20 \pm 7.83 \mathrm{sec}$ for the rapamycin-treated group $(\mathrm{P}<0.01 \mathrm{vs.}$. placebo group). The time-to-fall in the rapamycin-treated group was significantly longer compared with that in the MP-treated group $(\mathrm{P}<0.01$; Fig. 2E).

Assessment of plasma TGF- $\beta$ and IL-10 levels. To investigate the mechanisms by which rapamycin suppresses the immune response, the plasma levels of TGF- $\beta$ and IL-10 were subsequently measured, both of which are known to suppress immune responses (29). The plasma TGF- $\beta$ concentration was $2,087.00 \pm 74.27 \mathrm{pg} / \mathrm{ml}$ in the placebo group, $2,238.50 \pm 134.29 \mathrm{pg} / \mathrm{ml}$ in the MP-treated group, and $2,501.75 \pm 329.11 \mathrm{pg} / \mathrm{ml}$ in the rapamycin-treated group $(\mathrm{P}<0.05$ vs. placebo group; Fig. 3A). No significant differences were found between the placebo and MP-treated groups or between the MP- and rapamycin-treated groups $(\mathrm{P}>0.05$; Fig. 3A). The plasma IL-10 concentration was $15.36 \pm 0.50 \mathrm{pg} / \mathrm{ml}$ in the placebo group, $2.82 \pm 0.33 \mathrm{pg} / \mathrm{ml}$ in the MP-treated group ( $\mathrm{P}<0.01$ vs. placebo group), and $2.39 \pm 0.32 \mathrm{pg} / \mathrm{ml}$ in the rapamycin-treated group ( $\mathrm{P}<0.01$ vs. placebo group) (Fig. 3B). The plasma IL-10 levels were not significantly different between the MP-treated and the rapamycin-treated groups ( $\mathrm{P}>0.05$; Fig. 3B).

Treg cell frequency in the spleen. Finally, the frequency of splenic Treg cells was investigated in the three groups.
Splenocytes were analyzed using flow cytometry, and the percentages of Treg cells (Foxp3 ${ }^{+}$cells), calculated as the percentage of $\mathrm{CD} 4^{+} \mathrm{CD} 25^{+} \mathrm{Foxp}^{+}$cells out of $\mathrm{CD} 4^{+}$cells, were measured in all three groups (Fig. 4A-C). The percentage of splenic Treg cells was $15.6 \pm 1.71 \%$ in the placebo group, $8.9 \pm 0.83 \%$ in the MP-treated group ( $\mathrm{P}<0.05$ vs. placebo group), and $10.7 \pm 0.56 \%$ in the rapamycin-treated group $(\mathrm{P}<0.05$ vs. placebo group) (Fig. 4D). All three groups had significantly higher splenic Treg cell ratios compared with the normal mice (4.2\%; data not shown).

\section{Discussion}

In a previous study, a murine model of EAM was established to investigate the pathogenesis of autoimmune myositis (24). The present study further confirmed the validity of this model. Mice that underwent the EAM induction protocol exhibited symptoms that were typical of myositis, including a deteriorated body condition, muscle weakness, and inflammatory lesions in the muscles. Subsequently, the efficacy of MP and rapamycin for treating EAM was assessed, which demonstrated that treatment with rapamycin was significantly more beneficial compared with treatment with MP, in terms of improving the general body condition, muscle strength and alleviating inflammation. Since rapamycin is known to suppress the immune response by inhibiting mTOR, the effects of rapamycin on two key immune suppressive molecules, TGF- $\beta$ and IL-10, were compared with those of MP. The rapamycin-treated mice exhibited a significant increase in plasma TGF- $\beta$ levels, but not plasma IL-10 levels. Moreover, treatment with either MP or rapamycin was not associated with an increase in the proportion of splenic Treg cells. These results suggested that rapamycin 

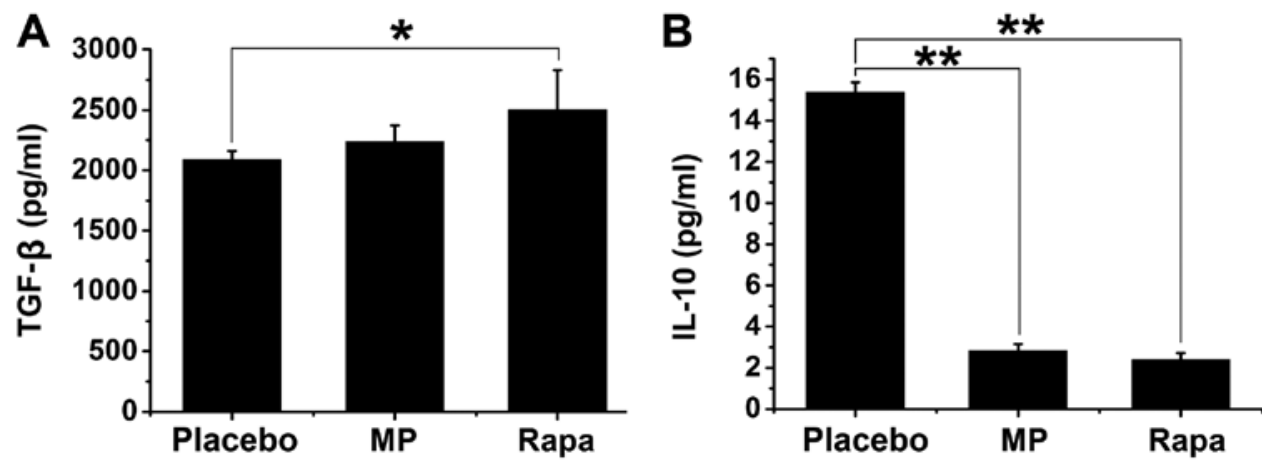

Figure 3. Treatment with Rapa and MP is associated with higher concentrations of plasma TGF- $\beta$ and lower concentrations of plasma IL-10 compared with the placebo group. At 15 days after initiation of the indicated treatments, blood samples were collected and plasma concentrations of (A) TGF- $\beta$ and (B) IL-10 were measured using the Luminex assay. $\mathrm{n}=6$ for each group. ${ }^{*} \mathrm{P}<0.05 ;{ }^{* *} \mathrm{P}<0.01$. MP, methylprednisolone, Rapa, rapamycin; TGF- $\beta$, transforming growth factor- $\beta$, IL-10, interleukin-10.
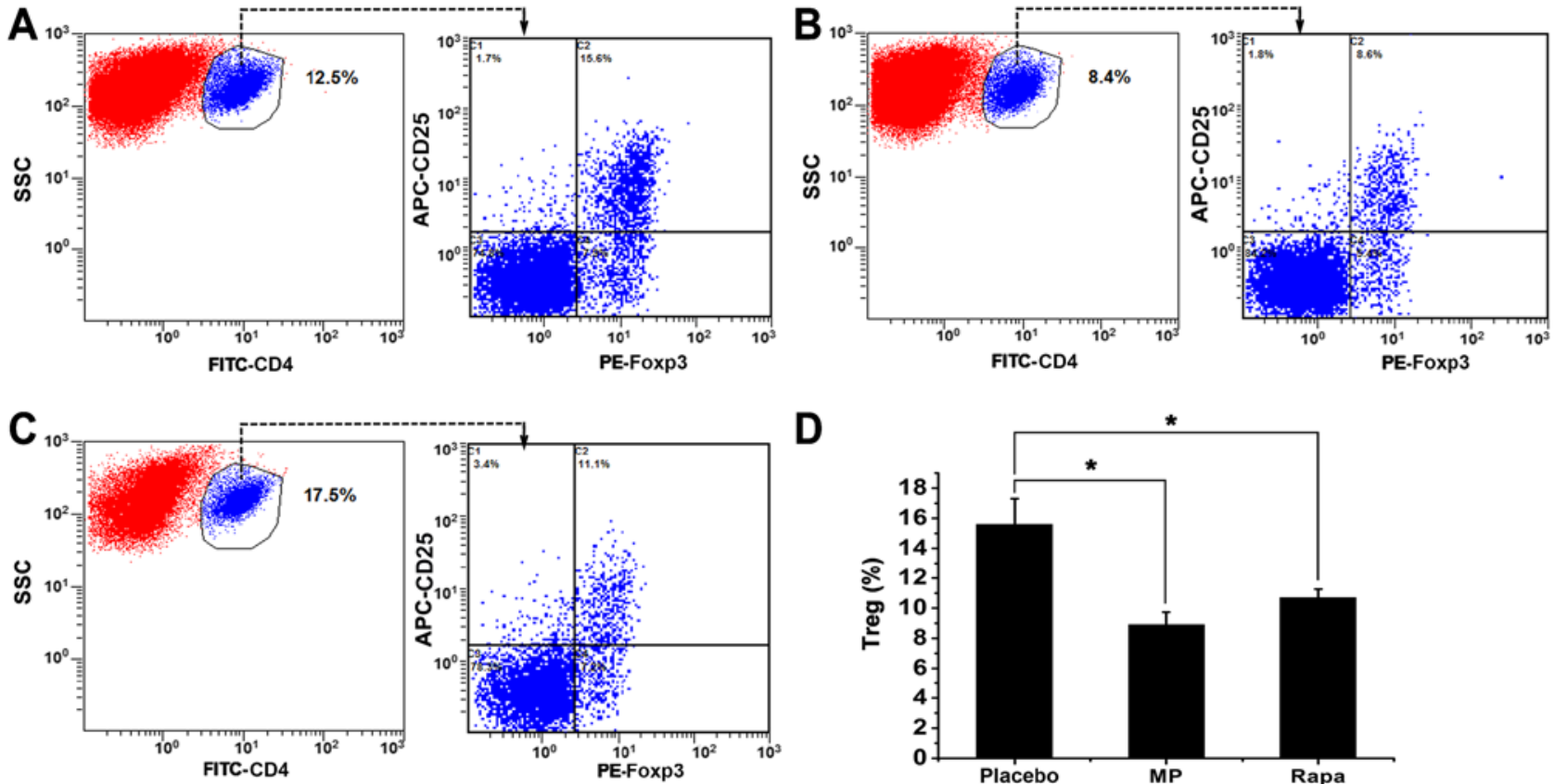

Figure 4. Percentage of splenic Treg cells in the placebo group is significantly higher compared to both the MP- and Rapa-treated groups. Representative flow profiles show the staining patterns of $\mathrm{CD} 4^{+} \mathrm{CD} 25^{+} \mathrm{Foxp} 3^{+}$Tregs in the spleens of EAM mice in (A) the placebo group, (B) the MP group and (C) the Rapa group at 15 days after initiation of treatment. Splenic cells were stained with FITC-CD4, APC-CD25 and PE-Foxp3. (D) Summary of the percentages of splenic Treg cells among total $\mathrm{CD}^{+} \mathrm{T}$ cells in the indicated groups. $\mathrm{n}=6$ for each group. ${ }^{*} \mathrm{P}<0.05$. Treg, regulatory $\mathrm{T}$ cells; EAM, experimental autoimmune myositis; MP, methylprednisolone; Rapa, rapamycin; PE, phycoerythrin; APC, allophycocyanin.

possibly exerts its anti-inflammatory effects via the TGF- $\beta$ signaling pathway.

Previous studies on the role of rapamycin in regulating TGF- $\beta$ expression have been inconsistent. Li et al (13) reported that rapamycin decreased the expression of TGF- $\beta$ in mononuclear cells in an autoimmune hepatitis model. Furthermore, in a rat model of unilateral ureteral obstruction, rapamycin was reported to decrease both the protein and mRNA expression of TGF- $\beta$ (14). In contrast, Yamane et al (30) reported that rapamycin promoted TGF- $\beta$ signaling and thereby increased ceramide synthesis in keratinocytes. In the present EAM model, treatment with rapamycin induced a moderate but significant increase in the plasma TGF- $\beta$ level. Moreover, rapamycin ameliorated inflammation in TGF- $\beta$ knockout mice (31), indicating a
TGF- $\beta$-independent mechanism of immunoregulation by rapamycin. In summary, it is likely that the modulation of the inflammatory response and TGF- $\beta$ signaling by rapamycin are context- and disease-dependent.

In the present study, it was found that the proportion of splenic Treg cells among total $\mathrm{CD}^{+} \mathrm{T}$ cells in normal mice was slightly lower than the reported normal range of $5-10 \%(32,33)$. Treg cells are known to counteract $\mathrm{T}$ cells that are responsible for muscle degradation (34). In a previous study on a murine model of EAM, the depletion of Treg cells was associated with more severe myositis, whereas the injection of expanded polyclonal Treg cells improved myositis (22). In addition, Treg cell frequency was inversely correlated with disease (35). Thus, the finding that the proportion of Treg cells was the highest in the placebo group was 
unexpected and inconsistent with a previous study, which found that rapamycin increased Treg frequency (23). Hence, as the placebo group of animals exhibited the most severe inflammation, it is possible that the higher ratio of Treg cells might represent elevated disease severity. Additionally, since the immune response is a complex process, multiple signaling pathways are often activated concurrently in response to the same stimuli (36). Furthermore, individual immune systems respond to the same immune insults differently, which adds another layer of complexity to the study of immune regulation. Therefore, further studies are necessary to fully understand the regulation and function of Treg cells in myositis.

The mTOR signaling pathway is a master regulator of cell growth and metabolism, and this pathway is activated downstream of the PI3K-AKT axis. The PI3K/AKT/mTOR pathway has been implicated in autoimmune diseases and cancer, and patients with these diseases can benefit from rapamycin treatment. For example, in a preclinical Dark Agouti rat model of multiple sclerosis, oral administration of rapamycin for 28 consecutive days significantly ameliorated protracted relapsing experimental allergic encephalomyelitis (11). Mammana et al (37) also reported that the PI3K/AKT/mTOR pathway was significantly involved in the etiopathogenesis of a murine model of multiple sclerosis, and that rapamycin treatment could be a potential therapeutic approach to treating multiple sclerosis clinically. Furthermore, the therapeutic potential of targeting the PI3K/AKT/mTOR pathway has also been highlighted in multiple malignancies, such as T-cell acute lymphoblastic leukemia (38). Since upregulated $\mathrm{PI} 3 \mathrm{~K} / \mathrm{AKT} / \mathrm{mTOR}$ signaling could also occur in inflammatory myopathies, a novel therapeutic approach consisting of dual inhibitors of the PI3K/AkT/mTOR pathway might be more potent than using rapamycin alone. For instance, NVP-BEZ235, an inhibitor of both PI3K and mTOR complex $1 / 2$, exhibited superior anti-cancer activity in an orthotopic bladder cancer model (39) Recently, p70S6 kinase, a downstream target of the PI3K/AKT/mTOR pathway, has gained attention for its potential as a therapeutic target for treating autoimmune diseases and cancer $(40,41)$. In addition, a class of nitric-oxide derivatives of antiretroviral protease inhibitors, such as Saquinavir-NO and Lopinavir-NO (42-44), can specifically inhibit p70S6 kinase, and is now under further investigation. Therefore, the results of the present study substantiate the superior efficacy of rapamycin in the mouse model of EAM, opening new avenues to study p70S kinase and its inhibitors in inflammatory muscle diseases.

TGF- $\beta$ is an anti-inflammatory cytokine and, consistent with the results of the present study, increased circulating levels of TGF- $\beta$ have been reported in patients treated with immunomodulatory drugs $(15,45)$. Nicoletti et al $(45)$ reported that TGF- $\beta$ levels were elevated in patients with both relapsing-remitting and chronic progressive multiple sclerosis, and treatment with interferon- $\beta$ augmented the increase in serum TGF- $\beta$ levels. Moreover, T cell cytokine profiling in patients with multiple sclerosis treated with rapamycin demonstrated a significant increase in serum TGF- $\beta$ levels (15). Taken together, TGF- $\beta$ induction might be a pharmacological mode of action by which rapamycin and other immunomodulators exert beneficial effects in inflammatory and autoimmune diseases. However, in the present study, only the impacts of rapamycin or MP on the plasma levels of TGF- $\beta$ and IL-10 in EAM mice were investigated; it is possible that other pro-inflammatory or anti-inflammatory cytokines might also be modulated by rapamycin. For example, the pro-inflammatory cytokine macrophage migration inhibitory factor (MIF) has been shown to activate the mTOR and AMP-activated protein kinase (AMPK) pathways in the pathogenesis of autoimmune diseases and cancer (46-49). Whether rapamycin treatment can also decrease the production of MIF to attenuate the activation of the PI3K/Akt/mTOR and AMPK pathways remains to be investigated.

In conclusion, using the established model of EAM, it was demonstrated that rapamycin had better efficacy than MP for treating myositis. Thus, the administration of rapamycin is a potential treatment option for patients with IIMs. However, since this is a preliminary in vivo animal study, the molecular signaling pathways involved in immunosuppression were not specifically examined. Further studies will be directed towards investigating the underlying molecular mechanisms of muscle inflammation and immunosuppression mediated by rapamycin.

\section{Acknowledgements}

Not applicable.

\section{Funding}

No funding was received.

\section{Availability of data and materials}

The datasets used and/or analyzed during the present study are available from the corresponding author on reasonable request.

\section{Authors' contributions}

JK and HJ conceived and designed the experiments; DF and FY performed the experiments; XT and WH analyzed the data; JK wrote the manuscript. All authors read and approved the final version of the manuscript.

\section{Ethics approval and consent to participate}

The experimental protocols were approved by the Institutional Animal Care and Use Committee of The Fourth Military Medical University. All animal procedures were in accordance with the ethical standards and practice of The Fourth Military Medical University.

\section{Patient consent for publication}

Not applicable.

\section{Competing interests}

The authors declare that they have no competing interests. 


\section{References}

1. Dalakas MC: Therapeutic advances and future prospects in immune-mediated inflammatory myopathies. Ther Adv Neurol Disord 1: 157-166, 2008.

2. Dalakas MC and Hohlfeld R: Polymyositis and dermatomyositis. Lancet 362: 971-982, 2003.

3. Hilton-Jones D: Inflammatory muscle diseases. Curr Opin Neurol 14: 591-596, 2001

4. Mastaglia FL and Phillips BA: Idiopathic inflammatory myopathies: Epidemiology, classification, and diagnostic criteria. Rheum Dis Clin North Am 28: 723-741, 2002.

5. Zeng L, Maruyama S, Nakamura K, Parker-Duffen JL, Adham IM, Zhong X, Lee HK, Querfurth $\mathrm{H}$ and Walsh K: The injury-induced myokine insulin-like 6 is protective in experimental autoimmune myositis. Skelet Muscle 4: 16 , 2014.

6. Kojima T, Tanuma N, Aikawa Y, Shin T, Sasaki A and Matsumoto Y: Myosin-Induced autoimmune polymyositis in the rat. J Neurol Sci 151: 141-148, 1997.

7. Gunawardena $\mathrm{H}$ : The clinical features of myositis-associated autoantibodies: A review. Clin Rev Allergy Immunol 52: 45-57, 2015.

8. Mozaffar T and Pestronk A: Myopathy with anti-Jo-1 antibodies: Pathology in perimysium and neighbouring muscle fibres. J Neurol Neurosurg Psychiatry 68: 472-478, 2000.

9. Gelardi C, Paolini L and Danieli MG: Subcutaneous immunoglobulin G in idiopathic inflammatory myopathies: Therapeutic implications. Isr Med Assoc J 16: 646-647, 2014.

10. Tournadre A: Therapeutic strategy in inflammatory myopathies (polymyositis, dermatomyositis, overlap myositis, and immune-mediated necrotizing myopathy). Rev Med Interne 35 466-471, 2014

11. Donia M, Mangano K, Amoroso A, Mazzarino MC, Imbesi R, Castrogiovanni P, Coco M, Meroni P and Nicoletti F: Treatment with rapamycin ameliorates clinical and histological signs of protracted relapsing experimental allergic encephalomyelitis in dark agouti rats and induces expansion of peripheral CD4+CD25+Foxp3+regulatory T cells.J Autoimmun 33: 135-140, 2009.

12. Gu L, Deng WS, Sun XF, Zhou H and Xu Q: Rapamycin ameliorates $\mathrm{CCl} 4$-induced liver fibrosis in mice through reciprocal regulation of the Th17/Treg cell balance. Mol Med Rep 14: 1153-1161, 2016

13. Li WW, Sun P, Chen DD, Wang WQ, Jiao GH, Wang YJ, Zhou L, Wang BM and Zhang J: Preventive and therapeutic effects of rapamycin against autoimmune hepatitis and liver fibrosis and possible mechanisms. Zhonghua Gan Zang Bing Za Zhi 24: 368-374, 2016 (In Chinese)

14. Liu CF, Liu H, Fang Y, Jiang SH, Zhu JM and Ding XQ: Rapamycin reduces renal hypoxia, interstitial inflammation and fibrosis in a rat model of unilateral ureteral obstruction. Clin Invest Med 37: E142, 2014.

15. Salehi M, Bagherpour B, Shayghannejad V, Mohebi F and Jafari R: Th1, Th2 and Th17 cytokine profile in patients with multiple sclerosis following treatment with rapamycin. Iran J Immunol 13: 141-147, 2016.

16. Wang B, Ding W, Zhang M, Li H and Gu Y: Rapamycin attenuates aldosterone-induced tubulointerstitial inflammation and fibrosis. Cell Physiol Biochem 35: 116-125, 2015.

17. Wang GY, Zhang Q, Yang Y, Chen WJ, Liu W, Jiang N and Chen GH: Rapamycin combined with allogenic immature dendritic cells selectively expands CD4+CD25+Foxp3+ regulatory T cells in rats. Hepatobiliary Pancreat Dis Int 11: 203-208, 2012.

18. Gambineri E, Torgerson TR and Ochs HD: Immune dysregulation, polyendocrinopathy, enteropathy, and X-linked inheritance (IPEX), a syndrome of systemic autoimmunity caused by mutations of FOXP3, a critical regulator of T-cell homeostasis. Curr Opin Rheumatol 15: 430-435, 2003.

19. Li X, Liang Y, LeBlanc M, Benner C and Zheng Y: Function of a Foxp3 cis-element in protecting regulatory $\mathrm{T}$ cell identity. Cell 158: 734-748, 2014

20. Shevach EM: Regulatory T cells in autoimmmunity. Ann Rev Immunol 18: 423-449, 2000.

21. Cohen JL, Trenado A, Vasey D, Klatzmann D and Salomon BL: CD4(+)CD25(+) immunoregulatory T cells: New therapeutics for graft-versus-host disease. J Exp Med 196: 401-406, 2002.
22. Allenbach Y, Solly S, Grégoire S, Dubourg O, Salomon B Butler-Browne G, Musset L, Herson S, Klatzmann D and Benveniste O: Role of regulatory T cells in a new mouse model of experimental autoimmune myositis. Am J Pathol 174: 989-998, 2009.

23. Prevel N, Allenbach Y, Klatzmann D, Salomon B and Benveniste $\mathrm{O}$ : Beneficial role of rapamycin in experimental autoimmune myositis. PLoS One 8: e74450, 2013.

24. Kang J, Zhang HY, Feng GD, Feng DY and Jia HG: Development of an improved animal model of experimental autoimmune myositis. Int J Clin Exp Pathol 8: 14457-14464, 2015.

25. Contet C, Rawlins JN and Deacon RM: A comparison of 129S2/SvHsd and C57BL/6JOlaHsd mice on a test battery assessing sensorimotor, affective and cognitive behaviours: Implications for the study of genetically modified mice. Behav Brain Res 124: 33-46, 2001.

26. Kohyama K and Matsumoto Y: C-Protein in the skeletal muscle induces severe autoimmune polymyositis in lewis rats. J Neuroimmunol 98: 130-135, 1999.

27. Matsumoto Y, Kohyama K, Park IK, Nakajima $M$ and Hiraki K: Characterization of pathogenic T cells and autoantibodies in C-protein-induced autoimmune polymyositis. J Neuroimmunol 190: 90-100, 2007.

28. Okiyama N, Hasegawa H, Oida T, Hirata S, Yokozeki H, Fujimoto M, Miyasaka N and Kohsaka H: Experimental myositis inducible with transfer of dendritic cells presenting a skeletal muscle C protein-derived CD8 epitope peptide. Int Immunol 27: 327-332, 2015.

29. Taylor A, Verhagen J, Blaser K, Akdis M and Akdis CA: Mechanisms of immune suppression by interleukin-10 and transforming growth factor-beta: The role of $\mathrm{T}$ regulatory cells. Immunology 117: 433-442, 2006.

30. Yamane T, Muramatsu A, Yoshino S, Matsui S, Shimura M, Tsujii Y, Iwatsuki K, Kobayashi-Hattori K and Oishi Y: MTOR inhibition by rapamycin increases ceramide synthesis by promoting transforming growth factor- $\beta 1 /$ Smad signaling in the skin. FEBS Open Bio 6: 317-325, 2016.

31. Borkowski TA, Letterio JJ, Farr AG and Udey MC: A role for endogenous transforming growth factor beta 1 in langerhans cell biology: The skin of transforming growth factor beta 1 null mice is devoid of epidermal langerhans cells. J Exp Med 184: 2417-2422, 1996.

32. Horwitz DA, Zheng SG and Gray JD: The role of the combination of IL-2 and TGF-beta or IL-10 in the generation and function of CD4+ CD25+ and CD8+ regulatory T cell subsets. J Leukoc Biol 74: 471-478, 2003.

33. Yagi H, Nomura T, Nakamura K, Yamazaki S, Kitawaki T, Hori S, Maeda M, Onodera M, Uchiyama T, Fujii S and Sakaguchi S: Crucial role of FOXP3 in the development and function of human CD25+CD4+ regulatory T cells. Int Immunol 16: 1643-1656, 2004.

34. Waschbisch A, Schwab N, Ruck T, Stenner MP and Wiendl H: FOXP3+ T regulatory cells in idiopathic inflammatory myopathies. J Neuroimmunol 225: 137-142, 2010.

35. Banica L, Besliu A, Pistol G, Stavaru C, Ionescu R, Forsea AM, Tanaseanu C, Dumitrache S, Otelea D, Tamsulea I, et al: Quantification and molecular characterization of regulatory T cells in connective tissue diseases. Autoimmunity 42: 41-49, 2009.

36. Mai J, Wang H and Yang XF: Th 17 cells interplay with Foxp3+ Tregs in regulation of inflammation and autoimmunity. Front Biosci (Landmark Ed) 15: 986-1006, 2010.

37. Mammana S, Bramanti P, Mazzon E, Cavalli E, Basile MS, Fagone P, Petralia MC, McCubrey JA, Nicoletti F and Mangano K: Preclinical evaluation of the PI3K/Akt/mTOR pathway in animal models of multiple sclerosis. Oncotarget 9: 8263-8277, 2018.

38. Evangelisti C, Evangelisti C, Chiarini F, Lonetti A, Buontempo F, Bressanin D, Cappellini A, Orsini E, McCubrey JA and Martelli AM: Therapeutic potential of targeting mTOR in T-cell acute lymphoblastic leukemia (review). Int J Oncol 45: 909-918, 2014.

39. Matsushima M, Kikuchi E, Matsumoto K, Hattori S, Takeda T, Kosaka T, Miyajima A and Oya M: Intravesical dual PI3K/mTOR complex $1 / 2$ inhibitor NVP-BEZ235 therapy in an orthotopic bladder cancer model. Int J Oncol 47: 377-383, 2015.

40. Pai C, Walsh CM and Fruman DA: Context-specific function of S6K2 in th cell differentiation. J Immunol 197: 3049-3058, 2016.

41. Babchia N, Calipel A, Mouriaux F, Faussat AM and Mascarelli F: The PI3K/Akt and mTOR/P70S6K signaling pathways in human uveal melanoma cells: Interaction with B-Raf/ERK. Invest Ophthalmol Vis Sci 51: 421-429, 2010 
42. Paskas S, Mazzon E, Basile MS, Cavalli E, Al-Abed Y, He M, Rakocevic S, Nicoletti F, Mijatovic S and Maksimovic-Ivanic D: Lopinavir-NO, a nitric oxide-releasing HIV protease inhibitor, suppresses the growth of melanoma cells in vitro and in vivo. Invest New Drugs 37: 1014-1028, 2019.

43. Basile MS, Mazzon E, Krajnovic T, Draca D, Cavalli E, Al-Abed Y, Bramanti P, Nicoletti F, Mijatovic S and Maksimovic-Ivanic D: Anticancer and differentiation properties of the nitric oxide derivative of lopinavir in human glioblastoma cells. Molecules 23: E2463, 2018.

44. Maksimovic-Ivanic D, Fagone P, McCubrey J, Bendtzen K, Mijatovic S and Nicoletti F: HIV-Protease inhibitors for the treatment of cancer: Repositioning HIV protease inhibitors while developing more potent NO-hybridized derivatives? Int J Cancer 140: 1713-1726, 2017.

45. Nicoletti F, Di Marco R, Patti F, Reggio E, Nicoletti A, Zaccone P, Stivala F, Meroni PL and Reggio A: Blood levels of transforming growth factor-beta 1 (TGF-beta1) are elevated in both relapsing remitting and chronic progressive multiple sclerosis (MS) patients and are further augmented by treatment with interferon-beta $1 \mathrm{~b}$ (IFN-beta1b). Clin Exp Immunol 113: 96-99, 1998.
46. Cui J, Zhang F, Wang Y, Liu J, Ming X, Hou J, Lv B, Fang S and Yu B: Macrophage migration inhibitory factor promotes cardiac stem cell proliferation and endothelial differentiation through the activation of the PI3K/Akt/mTOR and AMPK pathways. Int J Mol Med 37: 1299-1309, 2016.

47. Gunther S, Fagone P, Jalce G, Atanasov AG, Guignabert C and Nicoletti F: Role of MIF and D-DT in immune-inflammatory, autoimmune, and chronic respiratory diseases: From pathogenic factors to therapeutic targets. Drug Discov Today 24: 428-439, 2019.

48. Richard V, Kindt N and Saussez S: Macrophage migration inhibitory factor involvement in breast cancer (Review). Int J Oncol 47: 1627-1633, 2015.

49. Mangano K, Mazzon E, Basile MS, Di Marco R, Bramanti P, Mammana S, Petralia MC, Fagone P and Nicoletti F: Pathogenic role for macrophage migration inhibitory factor in glioblastoma and its targeting with specific inhibitors as novel tailored therapeutic approach. Oncotarget 9: 17951-17970, 2018.

This work is licensed under a Creative Commons Attribution-NonCommercial 4.0 International (CC BY-NC 4.0) License. 